\title{
Multiple rearrangements in cryptic species of electric knifefish, Gymnotus carapo (Gymnotidae, Gymnotiformes) revealed by chromosome painting
}

Cleusa Y Nagamachi*1,2, Julio C Pieczarka1,2, Susana SR Milhomem¹, Patricia CM O'Brien², Augusto CP de Souza ${ }^{3}$ and Malcolm A Ferguson-Smith²

\begin{abstract}
Background: Gymnotus (Gymnotidae, Gymnotiformes) is the Neotropical electric fish genus with the largest geographic distribution and the largest number of species, 33 of which have been validated. The diploid number varies from $2 n=39-40$ to $2 n=54$. Recently we studied the karyotype of morphologically indistinguishable samples from five populations of $G$. carapo sensu stricto from the Eastern Amazon of Brazil. We found two cytotypes, $2 n=42$ (30 M/SM + $12 \mathrm{ST} / \mathrm{A})$ and $2 \mathrm{n}=40(34 \mathrm{M} / \mathrm{SM}+6 \mathrm{ST} / \mathrm{A})$ and we concluded that the differences between the two cryptic species are due to pericentric inversions and one tandem fusion.

Results: In this study we use for the first time, whole chromosome probes prepared by FACS of the Gymnotus carapo sensu strictu species, cytotype with $2 n=42$. Using two color hybridizations we were able to distinguish pairs 1, 2, 3, 7, 9, $14,16,18,19,20$ and 21 . It was not possible to separate by FACS and distinguish each of the following chromosome pairs even with dual color FISH: $\{4,8\} ;\{10,11\} ;\{5,6,17\} ;\{12,13,15\}$. The FISH probes were then used in chromosome painting experiments on metaphases of the $2 n=40$ cytotype. While some chromosomes show conserved synteny, others are rearranged in different chromosomes. Eight syntenic associations were found.

Conclusions: These results show that the karyotype differences between these cryptic species are greater than assumed by classical cytogenetics. These data reinforce the previous supposition that these two cytotypes are different species, despite the absence of morphological differences. Additionally, the homology of repetitive DNA between the two provides evidence of recent speciation.
\end{abstract}

\section{Background}

Cross-species FISH using whole chromosome painting is widely used for phylogenomic studies in many vertebrate groups, including primates [1-7], bats [8,9], deer [10], birds [11,12], etc. These studies make important contributions to our understanding of genomic reorganization

* Correspondence: cleusanagamachi@pq.cnpq.br

1 Laboratório de Citogenética, Instituto de Ciências Biológicas, Universidade Federal do Pará, Belém, Pará, Brazil

Full list of author information is available at the end of the article and mechanisms of chromosome evolution in warmblooded vertebrates.

Research using chromosome painting in fishes is unusual. It has been used only with probes made by microdissection [13-19] or by CGH, the latter without defining chromosome pairs [20]. The probes made by flow cytometry have higher complexity than those made by microdissection, and are more appropriate for crossspecies hybridization [21]. However, there are no reports in the literature of FACS (Fluorescent Activated Chromo- 
some Sorting) generated probes for chromosome painting involving a whole fish genome.

The chromosome structure of a fish, a cold-blooded vertebrate, lacks both GC-rich and GC-poor compartments. The absence of compartmentalization of their genomes may be the reason for failure to obtain good Gbands (for revision, see [22]). Also, this could explain the difficulty in getting whole chromosome-specific probes by FACS. As well, fish chromosomes are small and there is not enough difference in size to allow the separation of each individual pair. An additional difficulty is to obtain fibroblast cultures and chromosome preparations of high quality and with sufficient metaphases.

Gymnotus (Gymnotidae - Gymnotiformes) is the Neotropical electric knifefish genus with the largest geographic distribution, occurring from southern Mexico to northern Argentina [23]. It is also the most diverse known Gymnotiformes genus, with 33 validated species, of which 18 are known to occur in the Amazon basin $[24,25,23,26]$. Previous cytogenetic studies show that the diploid number in this genus ranges from $2 n=39-40$ (with sex chromosomes of the type $\mathrm{X}_{1} \mathrm{X}_{2} \mathrm{Y}$ ) to $2 \mathrm{n}=54$, exhibiting variation in the karyotype formula, the quantity of heterochromatin, and the position of the Nucleolar Organization Region [27-30].

Gymnotus carapo (holotype from Suriname) was described by Linneaus in the first half of the XVIII century (Albert \& Crampton, 2003). Currently it is defined as G. carapo sensu stricto and is understood as a complex of morphologically similar or cryptic species, with a large distribution area (Albert \& Crampton, 2003). Cytogenetic studies of samples identified as G. carapo show different karyotypes: $2 \mathrm{n}=54$ and $2 \mathrm{n}=52$ from Southern Brazil, $2 \mathrm{n}$ $=48$ in Amazonas, 2n = 42 in Pará (reviewed [30]). Recently we studied the karyotype of morphologically indistinguishable individuals from five populations of $G$. carapo sensu stricto from the Eastern Amazon of Brazil. We found two cytotypes, $2 \mathrm{n}=42(30 \mathrm{M} / \mathrm{SM}+12 \mathrm{ST} / \mathrm{A})$ and $2 n=40(34 \mathrm{M} / \mathrm{SM}+6 \mathrm{ST} / \mathrm{A})$ and we concluded that the differences between these two cryptic species are due to pericentric inversions and one tandem fusion [31].

In this study we were able to use, for the first time, whole chromosome probes prepared by FACS from a fish, Gymnotus carapo sensu stricto species, with $2 \mathrm{n}=42$ cytotype. These probes were used in hybridizations on metaphases of the cytotype with $2 n=40$, to determine the differences between the karyotypes of the two cryptic species.

\section{Methods}

We performed chromosome painting analysis on two specimens of Gymnotus carapo from two localities in the Eastern Amazon Basin, Pará State, Brazil: Santa Cruz do Arari $\left(00^{\circ} 42^{\prime} 03.2^{\prime \prime} \mathrm{S}, 049^{\circ}, 10^{\prime} 42.1^{\prime \prime} \mathrm{W}\right)$ on Marajo Island
(MCP 40926) and Almeirim (01'31'34.2"S,052 33'37.9"W) (MPEG 13329). These specimens are part of the sample studied by Milhomem et al. [30,31].

A primary fibroblast cell line was established from an electric knifefish Gymnotus carapo sensu stricto, cytotype $2 \mathrm{n}=42$, from Santa Cruz do Arari (Marajo Island). Whole chromosome probes were made from this cell line at the Department of Veterinary Medicine, University of Cambridge, UK. The chromosome specific probes were made by degenerate oligonucleotide primed PCR (DOPPCR) on flow-sorted chromosomes as described previously $[32,10]$. Briefly, the chromosomes were prepared as described and stained with Hoechst $33258(2 \mathrm{mg} / \mathrm{mL})$ and Chromomycin A3 $(40 \mathrm{mg} / \mathrm{mL})$ in the presence of magnesium sulfate $(2.5 \mathrm{mmol} / \mathrm{L})$ for $2 \mathrm{~h}$. Sodium sulfate $(25 \mathrm{mmol} / \mathrm{L})$ and sodium citrate $(10 \mathrm{mmol} / \mathrm{L})$ were added 15 min prior to flow sorting. Chromosome sorting was performed using a dual-laser cell sorter (FACStar Plus; Becton Dickinson Immuno-Cytometry Systems). About 400 chromosomes were sorted from each peak in the flow karyotypes. Chromosomes were sorted directly into PCR tubes containing $30 \mu \mathrm{L}$ distilled water. These samples were amplified by DOP-PCR using the primer $6 \mathrm{MW}$ [32]. Primary PCR products were labelled either with biotin-16-dUTP (Boehringer Mannheim), Fluorescein isothiocyanate (FITC)-12-dUTP (Amersham) or Cy3-dUTP, by taking $1 \mu \mathrm{L}$ of product to a second round of DOP-PCR using the same primer. The biotin probes were detected with avidin-Cy3 or avidin-Cy5.

The metaphase chromosomal preparations from Gymnotus carapo sensu stricto, cytotype with $2 \mathrm{n}=40$ from Almeirim, were made following the methods described by Bertollo et al. [33].

In-situ hybridization of painting probes was performed as previously described [10]. Briefly, $14 \mu \mathrm{L}$ of the hybridization mixture (50\% formamide, $1 \times$ SSC, $10 \%$ dextran sulfate, $5 \mathrm{mg}$ salmon sperm DNA) and $1 \mu \mathrm{L}$ of labeled PCR product were denatured at $65^{\circ} \mathrm{C}$ for $1 \mathrm{~min}$. In-situ hybridization was performed for $48-72 \mathrm{~h}$ at $37^{\circ} \mathrm{C}$. The hybridization signal was detected as described earlier [10]. After hybridization and washing of the slides, biotinylated chromosome paints were detected with avidin (Vector Laboratories) coupled with Cy3, CY5 or FITC (Amersham). Probes directly linked to fluorochromes were also used, especially for dual or multi-FISH experiments. DAPI was used as a counterstain. In England, FISH digital images were obtained using a cooled CCD camera (Photometrics NU200 series equipped with a Kodak KAF 1400 chip) coupled to a Zeiss Axiophot microscope. The software Smart Capture VP (Digital Scientific) was used for camera control, digital image acquisition and the merging of DAPI and the fluorochrome images of the paints. In Brazil, digital images were captured with a CCD camera AxioCam Mrm coupled to a 


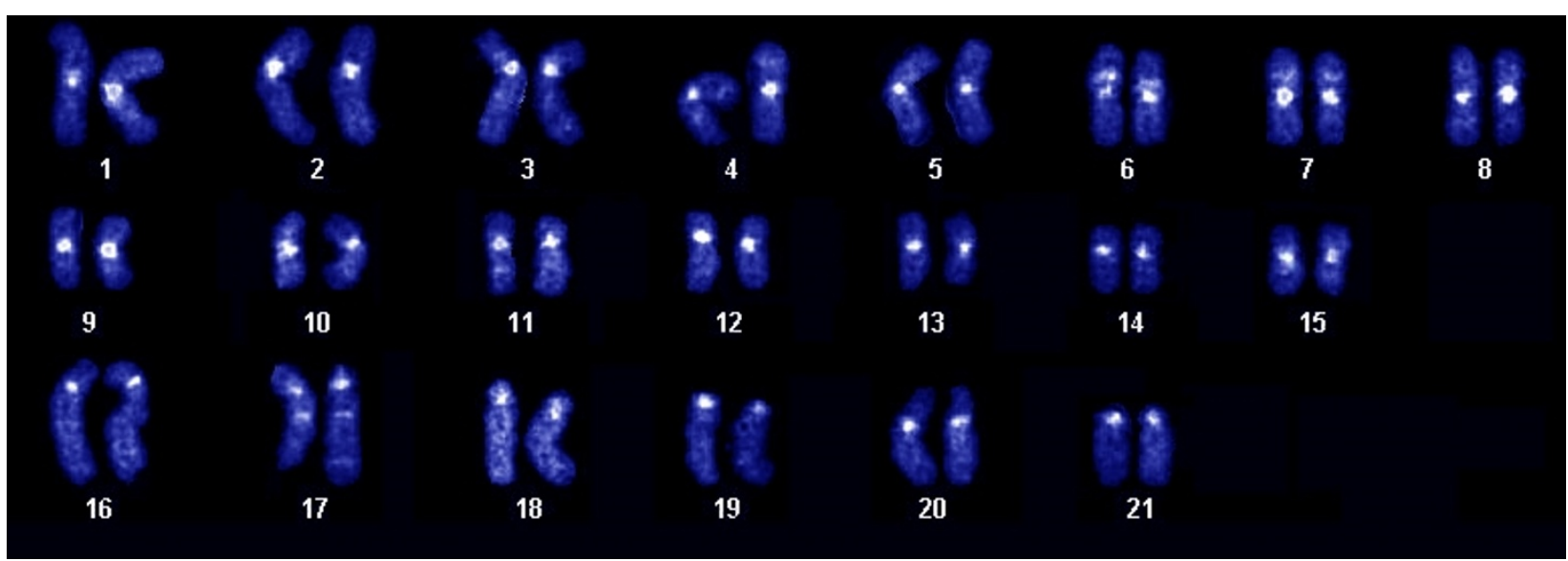

Figure 1 DAPI Karyotype of Gymnotus carapo sensu stricto: cytotype with $2 \mathrm{n}=42(30 \mathrm{M} / \mathrm{SM}+12 \mathrm{ST} / \mathrm{A})$.

Zeiss Axiophot microscope, using Axiovision 3.0 Software (Zeiss). The false color attribution was processed using Axiovision and the brightness and contrast corrected with Adobe Photoshop 7.1.

\section{Results}

Flow sorting of Gymnotus carapo sensu stricto, cytotype $2 \mathrm{n}=42$ : Characterization and chromosome identification.

Figure 1 shows the DAPI banding karyotype of Gymnotus carapo sensu stricto, cytotype with $2 \mathrm{n}=42(30 \mathrm{M} / \mathrm{SM}$ $+12 \mathrm{ST} / \mathrm{A})$, from which the whole chromosome paints were made. The bivariate flow karyotype shows the four regions (Figure 2), from which whole chromosome probes were produced. Hybridization experiments on metaphases from the same species (Figure 3) show that the only $\mathrm{GC}$-rich region, $\mathrm{R} 1$, is represented by the NORbearing chromosome (pair 20 - Figure 3a); R2 is represented by the four biggest pairs (1, 2, 3 and 16 - Figure $3 \mathrm{~b})$; R3 by the eight medium sized pairs $(4,5,6,7,8,17$, 18 and 19 - Figure 3c) and R4 by the eight smallest pairs $(9,10,11,12,13,14,15$ and 21 - Figure 3d). A same species multicolor FISH experiment with all the four probes shows that all the 42 chromosomes were hybridized,

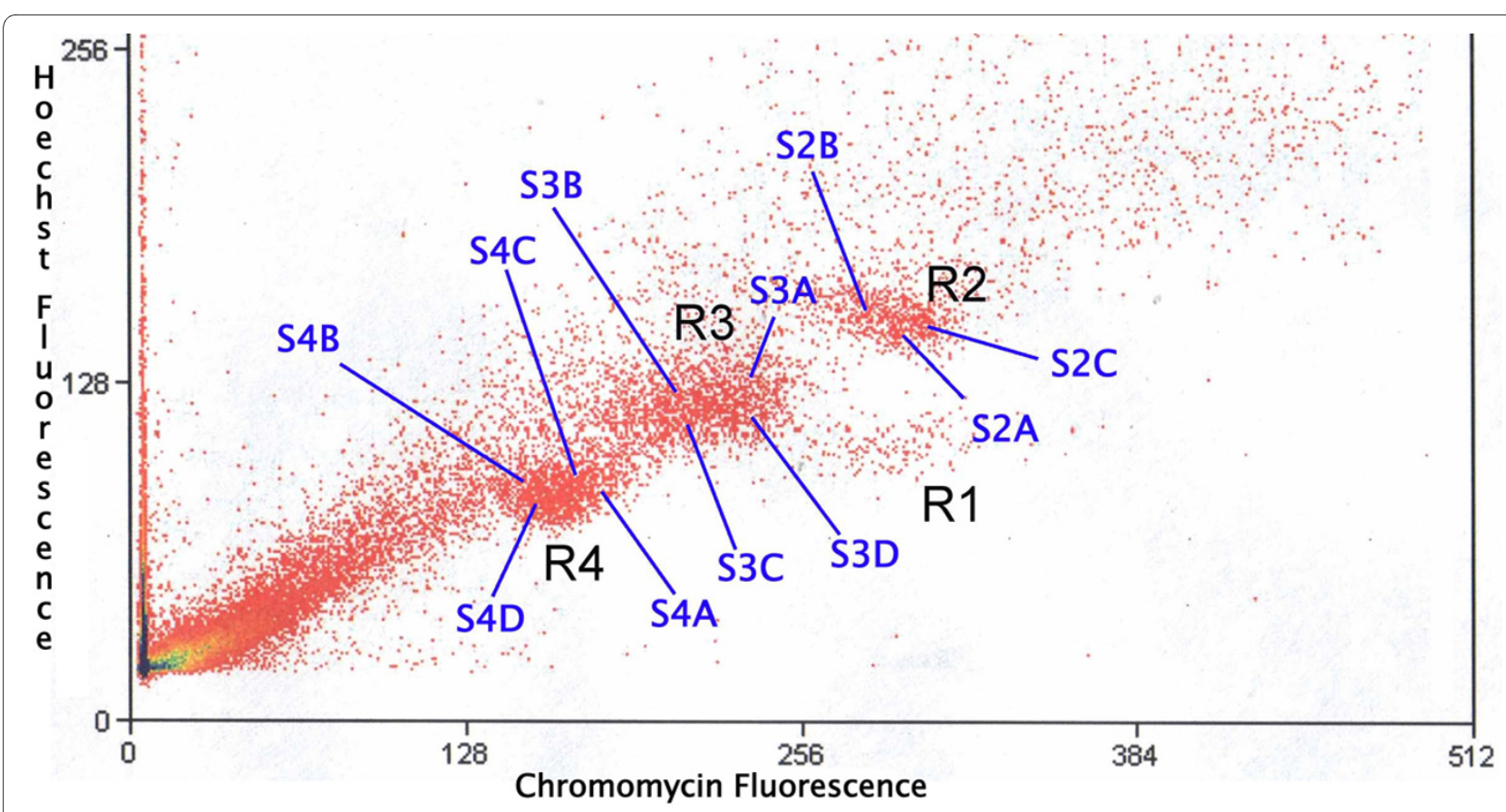

Figure 2 Bivariate flow karyotype showing 4 Regions: R1, R2 with 3 subregions (S2A, S2B, S2C), R3 with 4 subregions (S3A, S3B, S3C, S3D) and R4 with 4 subregions (S4A, S4B, S4C, S4D) 

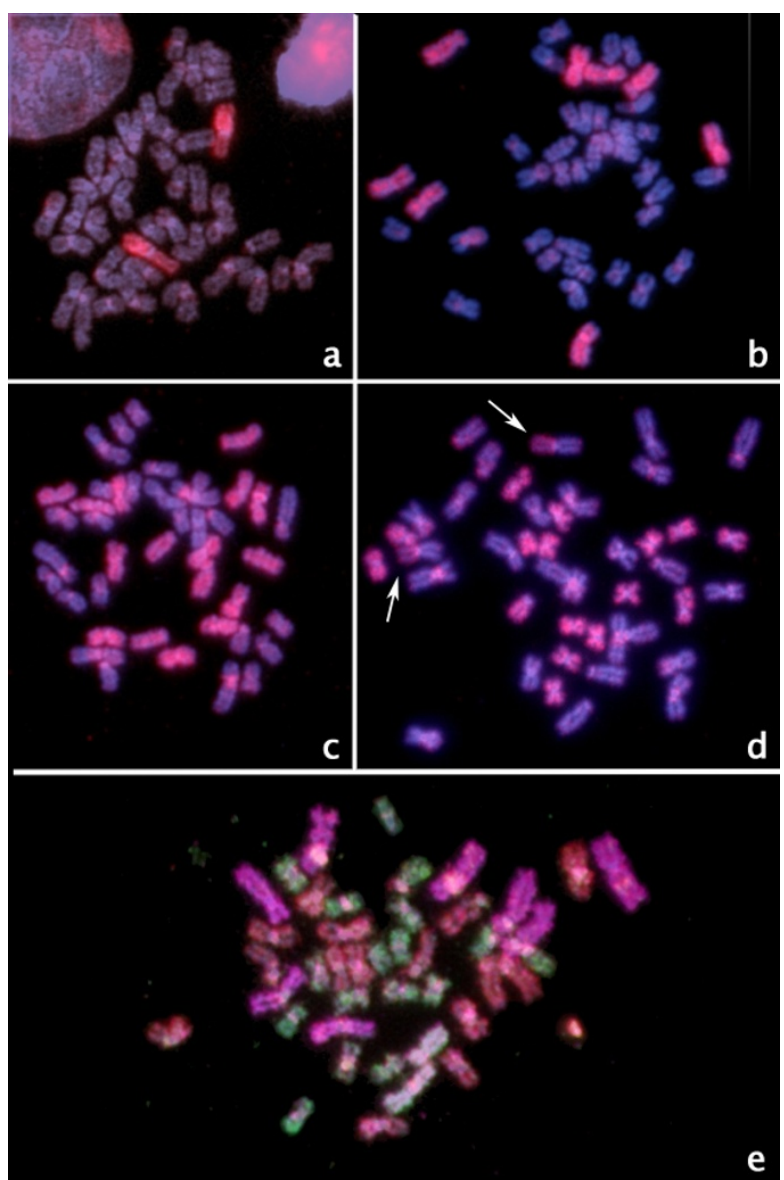

Figure 3 Hybridization experiments on same species metaphases: a) Region 1 (pair 20, NOR-bearing chromosome); b) Region 2 (pairs 1, 2, 3 and 16); c) Region 3 (pairs 4, 5, 6, 7, 8, 17, 18 and 19); d) Region 4 (pairs 9, 10, 11, 12, 13, 14, 15 and 21); arrows: NORs; e) Multicolor FISH with probes from the four regions: Region $\mathbf{1}$ = white; Region $\mathbf{2}$ = purple; Region $\mathbf{3}$ = red; Region $\mathbf{4}=$ green. All the chromosomes were counterstained with DAPI; probes (in red) from "a" to "d", stained with Cy3; 3e) red: Cy3; green: FITC; purple: Cy5; white: a mix of green and purple.

without superimposition (Figure 3e). From each of the three regions (R2, R3 and R4) further sorting produced three (R2) and four (R3 and R4) subregions (Figure 2). The results of same species hybridization showed that these subregions contain fewer chromosomes (Figure 4). From S2A, S2B, and S2C of R2: S2A contains pairs 1, 2 and 16; S2B contains pairs 2 and 16; S2C contains pairs 1 and 16. From S3A, S3B, S3C, and S3D of R3: S3A contains pairs 5, 6, 7 and 17; S3B contains pairs 7 and 19; S3C contains pair 7; S3D contains pairs 5, 6, 7, 17 and 18. From S4A, S4B, S4C, and S4D of R4: S4A contains pairs 12, 13 and 15; S4B contains pairs 12, 13, 14 and 15; S4C contains pairs 10, 11, 12, 13, 15 and 21; S4D contains pairs 12, 13, 14, 15 and 21. These results are summarized on Table 1. Apart from the homologous sequences, the probes also hybridized to non-specific regions, mainly in centro-

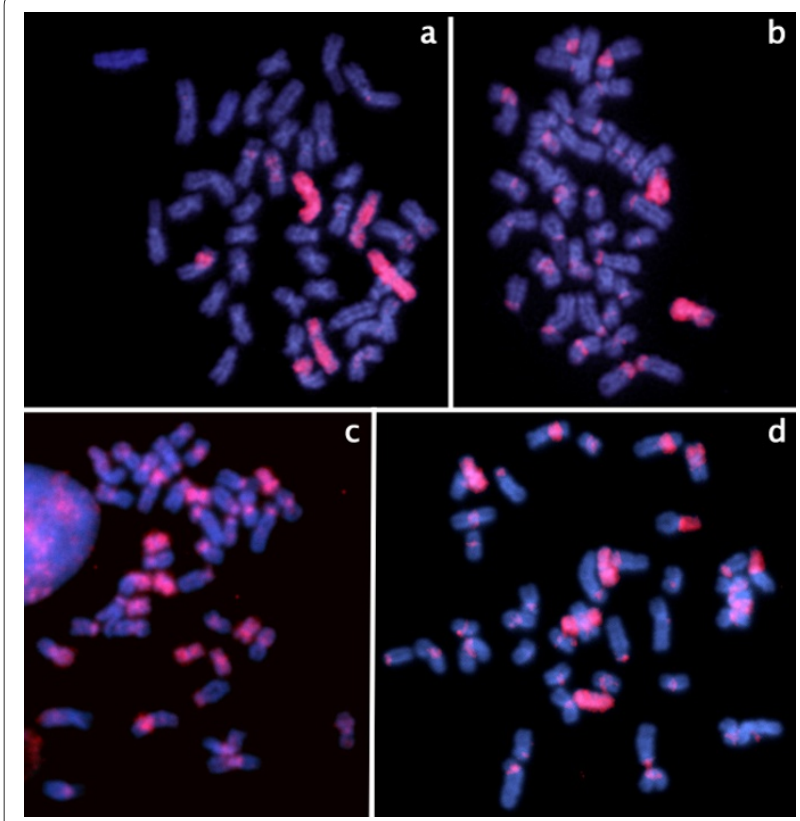

Figure 4 Examples of same species hybridization with subregions hybridizing fewer chromosomes: a) S2B (pairs 2 and 16); b) S3C (pair 7); C) S4B (pairs 9, 10, 15 and 21); d) S3B (pairs 7 and 19). All the chromosomes were counterstained with DAPI; probes stained with Cy3.

meric, interstitial, pericentromeric areas and/or the arms of some chromosomes, including the NOR. These areas have highly repetitive DNA.

Dual color hybridization was used for identification of chromosomes pairs from each of the regions (R2, R3 and $\mathrm{R} 4)$, as illustrated in Table 2 . In brief, it is possible to identify individually pairs 1, 2, 3, 7, 9, 14, 16, 18, 19, 20 and 21, while it was not possible to distinguish each of the following chromosome pairs even with dual color FISH: $\{4,8\}$; $\{10,11\} ;\{5,6,17\} ;\{12,13,15\}$.

Cross-species hybridization: Gymnotus carapo sensu stricto: cytotype $2 \mathrm{n}=42$ hybridized to cytotype $2 \mathrm{n}=40$

Whole chromosome paints from Gymnotus carapo sensu stricto, cytotype $2 \mathrm{n}=42$, were hybridized on metaphases of the cryptic species with cytotype $2 n=40$. For the precise definition of which chromosome belongs to each region, dual colour FISH was used with $\mathrm{R} 3 \times \mathrm{R} 4$ (Figure 5). It was possible to define the chromosome or chromosome segment in the $2 \mathrm{n}=40$ genome that corresponds to each chromosome from R3 (pairs 4, 5, 6, 7, 8, $17,18,19)$ and R4 (pairs 9, 10, 11, 12, 13, 14, 15, 21) from cytotype $2 n=42$. The remaining chromosome or chromosome segments correspond to R1 (the easily identified NOR pair - Figure 6a) or R2 (pairs 1, 2, 3, 16 - Figure 6b, c). Using dual color FISH with probes from the same region, it was possible to identify the chromosomes of this region (Figure 6). 
Table 1: Sorted regions of the flow karyotype with corresponding subregions and chromosome numbers.

\begin{tabular}{|c|c|c|c|c|}
\hline Region & $\begin{array}{c}\text { Number of } \\
\text { Chromosomes }\end{array}$ & $\begin{array}{c}\text { Region and } \\
\text { Subregion }\end{array}$ & Number of pairs & Chromosomes \\
\hline \multirow[t]{3}{*}{ R 1} & 1 & $\mathrm{R} 1$ & 1 pair & 20 \\
\hline & & $\mathrm{R} 2$ & 4 pairs & $1,2,3,16$ \\
\hline & & $\mathrm{S} 2 \mathrm{~A}$ & 3 pairs & $1,2,16$ \\
\hline \multirow[t]{3}{*}{$\mathrm{R} 2$} & 4 & $\mathrm{~S} 2 \mathrm{~B}$ & 2 pairs & 2,16 \\
\hline & & $\mathrm{S} 2 \mathrm{C}$ & 2 pairs & 1,16 \\
\hline & & R3 & 8 pairs & $4,5,6,7,8,17,18,19$ \\
\hline \multirow[t]{5}{*}{ R 3} & 8 & $\mathrm{~S} 3 \mathrm{~A}$ & 4 pairs & $5,6,7,17$ \\
\hline & & S3B & 2 pairs & 7,19 \\
\hline & & $\mathrm{S} 3 \mathrm{C}$ & 1 pair & 7 \\
\hline & & S3D & 5 pairs & $5,6,7,17,18$ \\
\hline & & R4 & 8 pairs & $9,10,11,12,13,14,15,21$ \\
\hline \multirow[t]{4}{*}{ R 4} & 8 & S4A & 3 pairs & $12,13,15$ \\
\hline & & S4B & 4 pairs & $12,13,14,15$ \\
\hline & & S4C & 6 pairs & $10,11,12,13,15,21$ \\
\hline & & S4D & 5 pairs & $12,13,14,15,21$ \\
\hline
\end{tabular}

Figure 7 shows the karyotype with DAPI banding of $G$. carapo $2 \mathrm{n}=40$ cytotype. Analysis of the hybridizations (Figure 7, Table 3) shows that the pairs 1, 2, 9, 14, 19, 20 and 21 (cytotype $2 \mathrm{n}=42$ ) had their synteny conserved and correspond to pairs 1, 2, 14, 17, 19, 20, 15 (cytotype $2 n=40$ ), respectively. Pairs $3,7,16$ and 18 were rearranged in two parts each. The $\{10,11\}$ group of chromosomes reveal two separate segments of homology, the $\{4,8\}$ group reveal three segments and the $\{5,6,17\}$ and the $\{12,13,15\}$ groups reveal four segments. The following syntenic associations were found (Figure 7, Table 3): 18/ $\{12,13,15\}$ (pair 3); $\{10,11\} / 3$ (pair 5); $3 /\{4,8\}$ (pair 6); 18/ 16 (pair 7); 7/\{5,6,17\} (pair 8); $7 /\{4,8\}$ (pair 9); $\{12,13,15\} /$ $\{10,11\}$ (pair 12); 16/\{5,6,17\} (pair 18).

The probes also painted highly repetitive DNA regions in the $2 n=40$ genome, usually in a pericentromeric region in a non-specific way. A large region on $4 \mathrm{p}$ proximal, 10p proximal and 11q proximal also painted in a non-specific way, probably because of highly repetitive DNA.

\section{Discussion}

Gymnotus carapo sensu stricto with 2n = 42 from Marajó Island (Pará-Brazil) is the first fish whose genome was used to generate FACS whole chromosome-specific probes covering the whole karyotype. This is a landmark in the cytogenetic studies of the family Gymnotidae (Gymnotiformes) and the fish in general.

The genome organization of fishes, with its small-sized chromosomes and non-compartmentalization of the chromosomes in GC- or AT-rich sequences (for review, see [22]), makes it difficult to isolate single chromosomes 
Table 2: Dual color FISH used to distinguish the chromosomes pairs of the Regions 2, 3 and 4.

\begin{tabular}{|c|c|}
\hline Dual color FISH & Chromosomes pairs \\
\hline & Region 2 \\
\hline $\mathrm{S} 2 \mathrm{~B} \times \mathrm{S} 2 \mathrm{C}$ & $\begin{array}{l}1 \text { (color of S2C); } 2 \text { (color of } \\
\text { S2B); } 16 \text { (two color) }\end{array}$ \\
\hline $\mathrm{R} 2 \times \mathrm{S} 2 \mathrm{~A}$ & $\begin{array}{c}3 \text { (color of R2); 1, 2, } 16 \text { (two } \\
\text { color) }\end{array}$ \\
\hline $\mathrm{R} 2 \times \mathrm{S} 2 \mathrm{~B}$ & $\begin{array}{c}\text { 2, } 16 \text { (two color); 1, } 3 \text { (color of } \\
\text { R2) - define pair } 3 \text { for } \\
\text { exclusion of pair } 1\end{array}$ \\
\hline
\end{tabular}

Region 3

\begin{tabular}{cc}
\hline S3B $\times$ S3C & 7 (two color); 19 (color of S3B) \\
\hline S3A $\times$ S3D & $\begin{array}{c}18 \text { (color of S3D); } 5,6,7,17 \\
\text { (two color) }\end{array}$ \\
\hline S3A $\times$ S3B & 7 (two color); 19 (color of \\
& S3B); $5,6,17$ (color of S3A) \\
\hline R3 $\times$ S3D & $5,6,7,17,18$ (two color); 4,8, \\
& 19 (color of R3) - define pairs \\
& 4,8 for exclusion of pair 19 \\
\end{tabular}

Region 4

\begin{tabular}{cc}
\hline S4A $\times$ S4B & $\begin{array}{c}14 \text { (color of S4B); 12, 13, 15 } \\
\text { (two color) }\end{array}$ \\
\hline R4 $\times$ S4C & $\begin{array}{c}9,14 \text { (color of R4); } 10,11,12, \\
13,15,21 \text { (two color) - define } \\
\text { pair } 9 \text { for exclusion of pair } 14\end{array}$ \\
\hline S4B $\times$ S4D & 21 (color of S4D); 12, 13, 14, \\
& 15 (two color) \\
\hline S4C $\times$ S4D & 14 (color of S4D); 10, 11 (color \\
& of S4C), 12, 13, 15, 21 (two \\
& color)
\end{tabular}

The dual color FISH allowed the distinction of all four chromosome pairs from Region 2. In Region 3, this allowed the distinction of pairs 7,18 and 19. It is not possible to distinguish pairs $\{4,8\}$ and $\{5,6,17\}$. In region 4 , this allowed the distinction of pairs 9,14 and 21 . It is not possible to distinguish pairs $\{10,11\}$ and $\{12,13,15\}$.

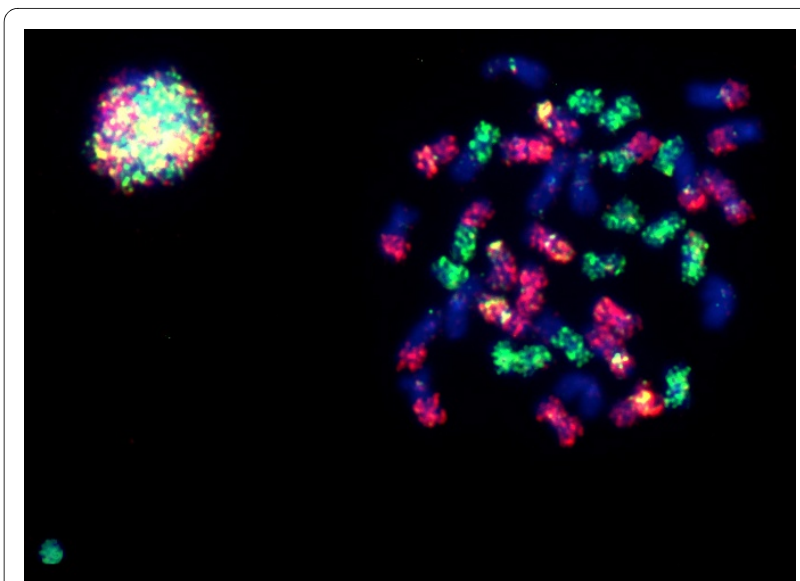

Figure 5 Cross species hybridization with R3 (red) and R4 (green) for the precise definition of which chromosome or chromosome segment in the $\mathbf{2 n}=\mathbf{4 0}$ genome corresponds to each chromosome from R3 (pairs 4, 5, 6, 7, 8, 17, 18, 19) and R4 (pairs 9, 10, 11, $12,13,14,15,21$ ) from cytotype $\mathbf{2 n}=\mathbf{4 2}$. All the chromosomes were counterstained with DAPI; red probes: Cy3; green probes: FITC.

by FACS. As a consequence, most of the probes obtained in our study represent chromosome groups and not single chromosomes. So, for chromosome identification we have to apply a strategy different from that used for warm-blooded vertebrates, in which most probes are actually derived from single chromosomes. Dual color FISH using probes from the same flow karyotype region allowed the identification of many chromosomes individually (pairs 1, 2, 3, 7, 9, 14, 16, 18, 19, 20, 21) except groups: $\{4,8\},\{10,11\},\{5,6,17\}$ and $\{12,13,15\}$, as shown in Table 2.

The mapping of these probes in the $2 \mathrm{n}=40$ G. carapo cytotype shows surprising and very interesting results. Using classical cytogenetics we supposed that the differences between these karyotypes resulted from a tandem fusion, to explain the diploid number difference, and several pericentric inversions to explain the differences in the karyotypic formula [31]. However, the cross-species FISH made clear that genomic rearrangements were much more frequent than supposed by classical cytogenetics. From the original 21 chromosome pairs of the $2 \mathrm{n}$ $=42$ G. carapo cytotype, only seven chromosomes (pairs $1,2,9,14,19,20,21$ ) have their synteny conserved in the $2 \mathrm{n}=40$ cytotype. The remaining chromosomes which were individually identified (pairs $3,7,16,18$ ) are all involved in rearrangements with other chromosomes (Figure 7, Table 3). From the chromosomes that remained in groups, the $\{4,8\}$ group of chromosomes paint a single chromosome (pair 10) and two other chromosomal segments (6q distal and 9q); the $\{5,6,17\}$ group of chromosomes paint two single chromosomes (pairs 4 and 11) and 


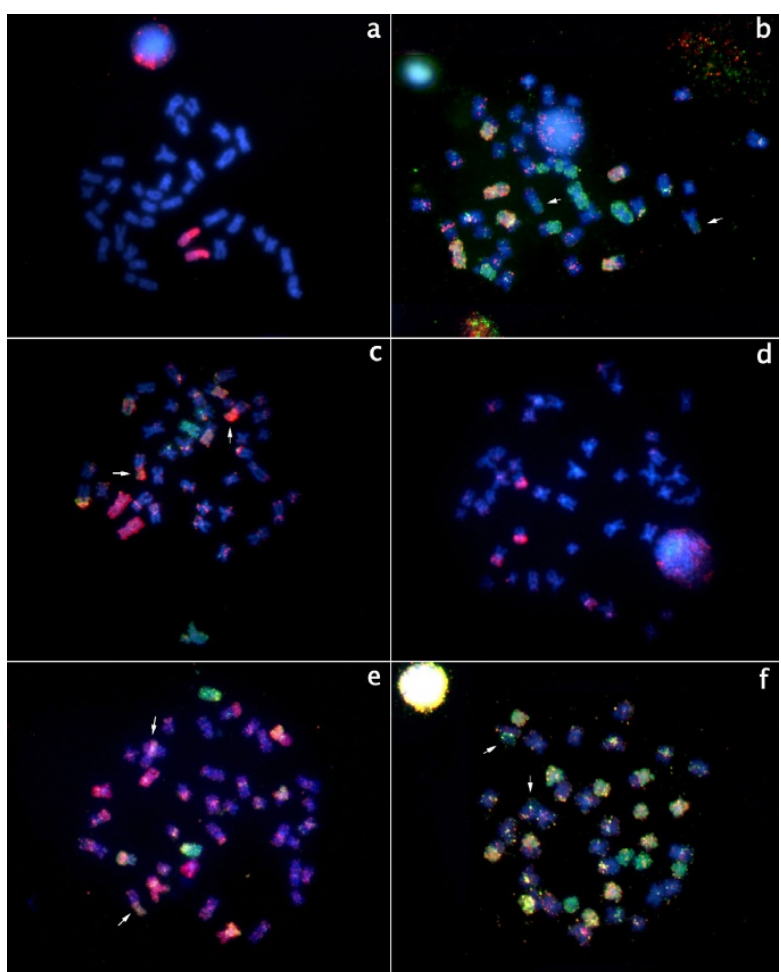

Figure 6 Examples of cross species hybridizations. Chromosome numbers refer to the original pairs in Gymnotus $2 n=42$ a) R1 (pair 20 red); b) S2B + R2 (pairs 1 and 3 green; pairs 2 and 16 yellow) C) S2B + S2C (pair 1 red, pair 2 green and pair 16 yellow) d) S3C (pair 7 red); e) S3A + S3B (pair 19 green, pair 7 yellow, pair $\{5,6,17\}$ red); f) S4C + R4 (pairs 9 and 14 green; pairs 10,11,12,13,15 and 21 yellow). Arrows on $\mathrm{b}, \mathrm{c}$, e and $\mathrm{f}$ point to the NOR region where nonspecific hybridizations occur. All the chromosomes were counterstained with DAPI; red probes: Cy3; green probes: FITC.

two segments (8q and $18 \mathrm{q}$ distal); the $\{10,11\}$ group of chromosomes paint two segments $(5 \mathrm{p}+\mathrm{q}$ proximal and $12 q)$; the $\{12,13,15\}$ group of chromosomes paint chromosomes 13 and 16 and two other chromosomal segments (3q and 12p).
These results show the great importance of comparative genomic mapping using FACS generated whole chromosome-specific probes. In cold-blooded vertebrates like fishes, whose chromosomes do not have a G-banding pattern, the number of rearrangements that differentiate karyotypes is likely to be underestimated. Probably many species with similar $2 \mathrm{n}$ and fundamental numbers have many rearrangements, like translocations, that do not change the diploid number and chromosome morphology.

The large genomic reorganization found between the two population cytotypes of Gymnotus carapo sensu stricto here studied confirms the hypothesis that they are really different species. The fact that the external morphology, the meristic data and their pigmentation do not allow their distinction [31], suggests that their speciation has been a recent event, in which chromosomal reorganization had a major role.

The paint probes from the $2 n=42$ genome also hybridize to highly repetitive DNA regions in the $2 \mathrm{n}=40$ genome, indicating that this DNA is homologous in both species. Normally, satellite DNA has great sequence divergence between closely related species, despite having great similarity within the repeats of one species [3436]. The homology of this DNA in both species is additional evidence for recent speciation.

The production of FACS whole chromosome probes from other fishes will be important for understanding chromosomal evolution in vertebrates. Certainly this approach is revealing a wealth of new data in these organisms.

\section{Conclusion}

The whole chromosome probes in Gymnotus carapo sensu stricto with $2 \mathrm{n}=42$ here described and obtained by FACS were used in a cross-species experiment. The chromosome painting demonstrated the large genomic reorganization found between the two cytotypes.

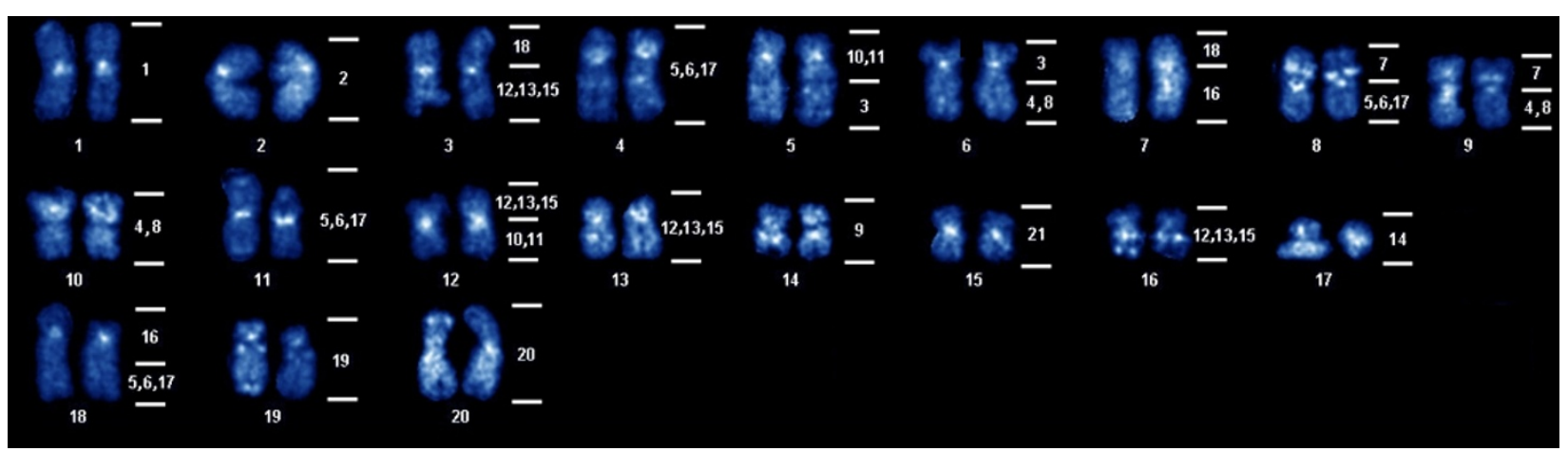

Figure 7 DAPI karyotype (mounted from the metaphase of Figure 5) of Gymnotus carapo sensu stricto, cytotype with $2 n=40$ ( 34 M/SM +6 ST/A) showing the results of comparative mapping with the chromosomes from the $2 n=42$ karyotype (numbers on the right side) 
Table 3: Comparative cross species analysis after the definition of groups 3 and 4 (Dual color FISH with R3 and R4 probes).

\begin{tabular}{|c|c|c|}
\hline Region & Pair of $2 n=42$ & Pair of $2 n=40$ \\
\hline \multirow[t]{2}{*}{ R 1} & 20 & 20 \\
\hline & 1 & 1 \\
\hline \multirow[t]{4}{*}{$\mathrm{R} 2$} & 2 & 2 \\
\hline & 3 & $5 q$ distal $+6(p+q$ prox. $)$ \\
\hline & 16 & $7 q+18$ (p+q prox.) \\
\hline & $\{4,8\}$ & $6 q$ distal $+9 q+10^{*}$ \\
\hline \multirow[t]{5}{*}{ R 3} & 7 & $8 p+9 p$ \\
\hline & $\{5,6,17\}$ & $4^{*}, 8 q, 11^{*}, 18 q$ distal \\
\hline & 18 & $3 p+7 p$ \\
\hline & 19 & 19 \\
\hline & 9 & 14 \\
\hline \multirow[t]{4}{*}{ R 4} & $\{10,11\}$ & $5 p+q$ prox., $12 q$ \\
\hline & $\{12,13,15\}$ & $3 q, 12 p, 13,16$ \\
\hline & 14 & 17 \\
\hline & 21 & 15 \\
\hline
\end{tabular}

\footnotetext{
${ }^{*}=$ chromosome pairs with non-specific hybridization on the heterochromatin
}

\section{Authors' contributions}

CYN conceived of the study, carried out chromosome painting in Gymnotus carapo sensu stricto with $2 n=42$ and $2 n=40$, organized the data and wrote most of the paper. JCP carried out chromosome painting in Gymnotus carapo sensu stricto with $2 \mathrm{n}=42$ and contributed to the discussion of data. SSRM carried out chromosome painting in Gymnotus carapo sensu stricto: cytotype $2 n=$ 40 and contributed to the discussion of data. ACPS collected the samples and helped with the FISH analysis. PCMO'B carried out the whole chromosome probes from Gymnotus carapo sensu stricto with $2 n=42$ cell lines. MAFS participated of the techniques development and coordinated the study. All authors read and approved the final manuscript.

\section{Acknowledgements}

We thank the Wellcome Trust for a grant to M. A. Ferguson-Smith and the CNPq-Brazil for the award of Post-Doctoral scholarships to Dr. Nagamachi and Dr. Pieczarka and a Doctoral scholarship to Susana SR Milhomem. We thank Dr. William GR Crampton for the taxonomic identification of the sample. Funding to the Brazilian authors was provided by CNPq, CAPES, UFPA, and FAPESPA. Collecting was authorized by IBAMA (Instituto Brasileiro do Meio Ambiente) permit 020/2005 (IBAMA Registration: 207419)

\section{Author Details}

1 Laboratório de Citogenética, Instituto de Ciências Biológicas, Universidade Federal do Pará, Belém, Pará, Brazil, ${ }^{2}$ Cambridge Resource Centre for Comparative Genomics, Department of Veterinary Medicine, University of Cambridge, Cambridge, UK and ${ }^{3}$ Centro Federal de Educação Tecnológica, Belem, Brazil

Received: 9 November 2009 Accepted: 27 April 2010

Published: 27 April 2010

\section{References}

1. Jauch A, Wienberg J, Stanyon R, Arnold N, Tofanelli S, Ishida T, Cremer T: Reconstruction of genomic rearrangements in great apes and gibbons by chromosome painting. Proceedings of the National Academy of Sciences USA 1992, 89:8611-8615.

2. Wienberg J, Stanyon R, Jauch A, Cremer T: Homologies in human and Macaca fuscata chromosomes revealed by in situ suppression hybridization with human chromosome specific DNA libraries. Chromosoma 1992, 101:265-270. 
3. Koehler U, Bigoni F, Wienberg J, Stanyon R: Genomic reorganization in the concolor gibbon (Hylobates concolor) revealed by chromosome painting. Genomics 1995, 30:287-292.

4. Neusser M, Stanyon R, Bigoni F, Wienberg J, Müller S: Molecular cytotaxonomy of New World Monkeys (Platyhrrini). Comparative analysis of five species by multicolor chromosome painting gives evidence for a classification of Callimico goeldii within the family of Callitrichidae. Cytogenetics and Cell Genetics 2001, 94:206-215.

5. Barros RMS, Nagamachi CY, Pieczarka JC, Rodriques LRR, Neusser M, De Oliveira EHC, Wienberg J, Muniz JAPC, Rissino JD, Muller S: Chromosomal studies in Callicebus donacophilus pallescens, with classic and molecular cytogenetic approaches: Multicolour FISH using human and Saguinus oedipus painting probes. Chromosome Research 2003, 11:327-334.

6. De Oliveira EHC, Neusser M, Pieczarka JC, Nagamachi CY, Sbalqueiro IJ, Muller S: Phylogenetic inferences of Atelinae (Platyrrhini) based on multi-directional chromosome painting in Brachyteles arachnoides, Ateles paniscus paniscus and Ateles b. marginatus. Cytogenetic and Genome Research 2005, 108:183-190.

7. Amaral PJS, Finotelo LFM, De Oliveira EHC, Pissinatti A, Nagamachi CY, Pieczarka JC: Phylogenetic studies of the genus Cebus (CebidaePrimates) using chromosome painting and G-banding. BMC Evolutionary Biology 2008, 8:169.

8. Pieczarka JC, Nagamachi CY, O'Brien PCM, Yang F, Rens W, Barros RMS, Noronha RCR, Rissino J, De Oliveira EHC, Ferguson-Smith MA: Reciprocal chromosome painting between two South American bats: Carollia brevicauda and Phyllostomus hastatus (Phyllostomidae, Chiroptera). Chromosome Research 2005, 13:339-347.

9. Ao L, Gu X, Feng Q, Wang J, O'Brien PCM, Fu B, Mao X, Su W, Wang Y, Volleth M, Yang F, Nie W: Karyotype relationships of six bat species (Chiroptera, Vespertilionidae) from China revealed by chromosome painting and G-banding comparison. Cytogenetic and Genome Research 2006, 115:145-153.

10. Yang F, Carter NP, Shi L, Ferguson-Smith MA: A comparative study of karyotypes of muntjacs by chromosome painting. Chromosoma 1995, 103:642-652

11. Guttenbach M, Nanda I, Feichtinger W, Masabanda JS, Griffin DK, Schmid M: Comparative chromosome painting of chicken autosomal paints 1 9 in nine different bird species. Cytogenetic and Genome Research 2003, 103:173-184.

12. De Oliveira EHC, de Moura SP, dos Anjos LJS, Nagamachi CY, Pieczarka JC, O'Brien PCM, Ferguson-Smith MA: Comparative chromosome painting between chicken and spectacled owl (Pulsatrix perspicillata): implications for chromosomal evolution in the Strigidae (Aves, Strigiformes). Cytogenetic and Genome Research 2008, 122:157-162.

13. Reed KM, Bohlander SK, Phillips RB: Microdissection of the $Y$ chromosome and fluorescence in situ hybridization analysis of the sex chromosomes of lake trout, Salvelinus namaycush. Chromosome Research 1995, 3:221-226.

14. Campos-Ramos R, Harvey SC, Masabanda JS, Carrasco LA, Griffin DK, et al:: Identification of putative sex chromosomes in the blue tilapia, Oreochromis aureus, through synaptonemal complex and FISH analysis. Genetica 2001, 111:143-153.

15. Phillips RB, Konkol NR, Reed KM, Stein JD: Chromosome painting supports lack of homology among sex chromosomes in Oncorhynchus, Salmo, and Salvelinus (Salmonidae). Genetica 2001, 111:119-123.

16. Harvey SC, Masabanda J, Carrasco LA, Bromage NR, Penman DJ, Griffin DK: Molecular-cytogenetic analysis reveals sequence differences between the sex chromosomes of Oreochromis niloticus: evidence for an early stage of sex-chromosome differentiation. Cytogenetic and Genome Research 2002, 97(1-2):76-80

17. Liu JD, Yi MS, Zhao G, Zhou F, Wang DQ Yu OX: Sex chromosomes in the spiny eel (Mastacembelus aculeatus) revealed by mitotic and meiotic analysis. Cytogenetic and Genome Research 2002, 98:291-297.

18. Henning F, Trifonov V, Ferguson-Smith MA, de Almeida-Toledo LF: Nonhomologous sex chromosomes in two species of the genus Eigenmannia (Teleostei: Gymnotiformes). Cytogenetic and Genome Research 2008, 121:55-58.

19. Henning F, Trifonov V, de Almeida-Toledo LF: Use of chromosome microdissection in fish molecular cytogenetics. Genetics and Molecular Biology 2008, 31(1 suppl):279-283.
20. Traut $\mathrm{W}$, Winking $\mathrm{H}$ : Meiotic chromosomes and stages of sex chromosome evolution in fish: zebrafish, platyfish and guppy. Chromosome Research 2001, 9(8):659-672.

21. Ferguson-Smith MA, Yang F, O'Brien PCM: Comparative mapping using chromosome sorting and painting. ILAR Journal 1998, 39(2-3):68-76.

22. Sharma OP, Tripathi NK, Sharma KK: A review of chromosome banding in fishes. In Some aspects of chromosome structure and function Edited by: Sobti RC, Obe G, Athwal RS. Narosa Publishing House, New Delhi, India; 2002:109-122.

23. Albert JS, Crampton WGR, Thorsen DH, Lovejoy NR: Phylogenetic systematics and historical biogeography of the Neotropical electric fish Gymnotus (Teleostei: Gymnotidae). Systematics and Biodiversity 2005, 2(4):375-417.

24. Albert JS, Crampton WGR: Five new species of Gymnotus (Teleostei: Gymnotiformes) from an Upper Amazon floodplain, with descriptions of electric organ discharges and ecology. Ichthyological Exploration of Freshwaters 2001, 12(3):241-266

25. Albert JS, Crampton WGR: Seven new species of the Neotropical electric fish Gymnotus (Teleostei, Gymnotiformes) with a redescription of $G$. carapo (Linnaeus). Zootaxa 2003, 28:1-54.

26. Crampton WGR, Thorsen DH, Albert JS: Three new species from a diverse, sympatric assemblage of the electric fish Gymnotus (Gymnotiformes: Gymnotidae) in the lowland Amazon Basin, with notes on ecology. Copeia 2005, 1:82-99.

27. Foresti F, Almeida-Toledo LF, Toledo-Filho SA: Chromosome studies in Gymnotus carapo and Gymnotus sp. (Pisces, Gymnotidae). Caryologia 1984, 37:141-146.

28. Sánchez S, Laudicina A, Jorge LC: A new report of multiple sex chromosome system in the Order Gymnotiformes (Pisces). Cytologia 2004, 69:155-160

29. Silva EB, Margarido VP: An X1X1X2X2/X1X2Y multiple sex chromosome system in a new species of the genus Gymnotus (Pisces, Gymnotiformes). Environmental Biology of Fishes 2005, 73:293-297.

30. Milhomem SSR, Pieczarka JC, Crampton WGR, de Souza ACP, Carvalho JR, Nagamachi CY: Differences in karyotype between two sympatric species of Gymnotus (Gymnotiformes: Gymnotidae) from the Eastern Amazon of Brazil. Zootaxa 2007, 1397:55-62

31. Milhomem SSR, Pieczarka JC, Crampton WGR, Silva DS, De Souza ACP, Carvalho JR Jr, Nagamachi CY: Chromosomal evidence for a putative cryptic species in the Gymnotus carapo species-complex (Gymnotiformes, Gymnotidae). BMC Genetics 2008, 9:75.

32. Telenius H, Pelmear A, Tunnacliffe A, et al: Cytogenetic analysis by chromosome painting using DOP-PCR amplified flow-sorted chromosomes. Genes Chromosomes Câncer 1992, 4:257-263.

33. Bertollo LAC, Takashi CS, Moreira-Filho O: Cytotaxonomic considerations on Hoplias lacerdae (Pisces, Erythrinidae). Brazilian Journal of Genetics 1978, 1(2):103-120.

34. Dover GA: Molecular drive: a cohesive mode of species evolution. Nature 1982, 299:111-117.

35. Grenier E, Castagnone-Sereno P, Abad P: Satellite DNA sequences as taxonomic markers in nematodes of agronomic interest. Parasitology Today 1997, 13(10):398-401

36. Abadon M, Grenier E, Laumond C, Abad P: A species-specific satellite DNA from the entomopathogenic nématode Heterorhabditis indicus. Genome 1998, 41:148-153.

doi: 10.1186/1471-2156-11-28

Cite this article as: Nagamachi et al., Multiple rearrangements in cryptic species of electric knifefish, Gymnotus carapo (Gymnotidae, Gymnotiformes) revealed by chromosome painting BMC Genetics 2010, 11:28 